\title{
Factores asociados a la satisfacción vital en adultos mayores de 60 años
}

\author{
Mauricio Ramírez Pérez
}

Universidad de Tarapacá, Arica, Chile. Email: mramirezp@achs.cl.

\author{
Sau-Lyn Lee Maturana \\ Universidad de Tarapacá, Arica, Chile. Email: slee@uta.cl.
}

\begin{abstract}
Resumen: Para la ejecución de este trabajo se entrevistaron a 122 adultos mayores de 60 años de la ciudad de Arica (52,5\% hombres y 47,5\% mujeres). A éstos se les administró un cuestionario de Satisfacción Vital SWLS (Diener, Emmons, Larsen y Griffin, 1985) junto con un cuestionario demográfico, para determinar cuáles de estas variables de contexto por si solas o en su conjunto, explican la Satisfacción Vital. El objetivo principal de este estudio es evidenciar cómo los indicadores de la Satisfacción Vital, se ven influidas por la situación de pareja comparando las puntuaciones medias del cuestionario.
\end{abstract}

Palabras clave: Satisfacción Vital, Adultos Mayores, Variables Sociodemográficas.

\section{Factors associated to life satisfaction in adults over 60 years old}

\begin{abstract}
To carry out this study 122 people over 60 years old were interviewed inhabitants of the city of Arica (52,5\% men and 47,5\% women). They answered the Vital Satisfaction questionnaire SWLS (Diener, Emmons, Larsen and Griffin, 1985), along with a demographic questionnaire to determine which of these variables of context by themselves or in in their totality, explain Vital Satisfaction. The main objective of this study is to demonstrate how indicators of Vital Satisfaction are influenced by the situation of their life with their mates, when comparing the average scores of the questionnaire. bles.

Key words: Vital Satisfaction, Old people, social and Demographic Varia-
\end{abstract}

\section{Fatores associados à satisfação com a vida em adultos acima de 60 anos}

Resumo: Para realizar este trabalho, entrevistou-se 122 adultos com mais de 60 anos de Arica (52,5\% homens e 47,5\% mulheres). Estes receberam um questionário sobre satisfação com a vida SWLS (Diener, Emmons, Larsen \& Griffin, 1985), juntamente com um questionário demográfico, para determinar qual dessas variáveis _ de contexto, por si ou em conjunto, explicam satisfação com a vida. $\mathrm{O}$ principal objetivo deste estudo é mostrar como indicadores de satisfação com a vida, são influenciados pela situação dos casais comparando os valores médios do questionário. 
Palavras-chave: satisfação com a vida, o envelhecimento, as variáveis sociodemográficas.

$$
* * *
$$

\section{Introducción}

Una de las cuestiones más características y distintivas del ser humano es su capacidad para valorar su calidad de vida (Dávila y Díaz, 2005). En este sentido, Veenhoven (1984) y Cole, Peeke y Dolezal (1999), señalan que la evaluación que las personas hacen de sus vidas, incluye una dimensión cognitiva (referida a la satisfacción con la vida en su totalidad o la satisfacción por áreas específicas como la matrimonial, laboral, etc.) y otra afectiva (relacionada con la frecuencia e intensidad de las emociones positivas y negativas), cuya interacción abarca un amplio espectro de vivencias. Esta autoevaluación del hombre claramente ha estado presente desde siempre, sin embargo, como señalan Cuadra y Florenzano (2003), tradicionalmente la psicología se ha orientado hacia el estudio y comprensión de las patologías y las enfermedades mentales. Este enfoque de la psicología clásica según Seligman y Csikszentmihalyi (2000), la ha hecho descuidar los aspectos positivos, tales como el bienestar, el contento, la satisfacción, la esperanza, el optimismo, el flujo y la felicidad, ignorándose los beneficios que éstos presentan para las personas.

Esta situación según lo plantean Diener, Suh, Lucas y Smith (1999) ha cambiado en los últimos treinta años ya que se ha incrementado considerablemente el número de investigaciones relacionadas con los diferentes aspectos del bienestar subjetivo. Goleman (2003) señala de forma clara que sólo muy recientemente, la psicología ha emprendido de manera explícita programas para el estudio de los aspectos positivos de la naturaleza humana. Comienza a advertirse la presencia de un movimiento en ciernes hacia lo que se denomina "psicología positiva", es decir, la investigación científica del bienestar y de las cualidades más positivas del ser humano. Existe un trabajo con ahínco por parte de los psicólogos en predecir la satisfacción vital y la felicidad, por medio del empleo de diferentes variables de predicción (Cornejo y Lucero, 2005; Zhang, 2005). La felicidad, que ha sido interpretada por filósofos y escritores de todas las épocas, incluyendo aquello de que "la felicidad no existe”, posiblemente por su concepción sublime y extrema, ha sido retomada por la psicología con una fórmula sencilla y operativa con el nombre de Bienestar Subjetivo (Hernández, 2006). Así, queda en evidencia que ha existido un auge en estas investigaciones que tratan en cierta medida de "compensar" este descuido, abordando desde distintos puntos de vista la explicación para el bienestar de las personas, es por esto que el presente estudio busca ser un aporte en la explicación de la Satisfacción Vital en una muestra con características particulares. 


\section{Satisfacción Vital}

El estudio del bienestar ha sido tema de interés de diversas disciplinas y campos de la psicología y se ha relacionado con felicidad, calidad de vida y salud mental, así como con distintas variables personales y contextuales asociadas (Ballesteros, Medina, Caicedo, 2006). Blanco y Díaz (2005) señalan que el estudio del bienestar en el seno de la Psicología se remonta a la década de los sesenta y en su transcurso ha dado lugar a dos grandes tradiciones, la "Hedónica" representada por una sólida línea de investigaciones que se ha ocupado del estudio del bienestar subjetivo, y la tradición "Eudaimónica”, centrada en el bienestar psicológico. Ryan y Deci (2001) ahondan en esta diferenciación señalando que la perspectiva Hedónica implica satisfacción con la vida, la presencia de estado de ánimo positivo y ausencia de estado de ánimo negativo (cuyo conjunto determina la felicidad); también incluye el valor asignado a las metas, el cual es individual y específico en cada cultura. Por su parte, las perspectivas Eudaimónicas han diferenciado la felicidad del bienestar y proponen que lograr las cosas valoradas no siempre lleva a obtener bienestar, independientemente del placer que produzca ese logro. Incluyen en su definición la realización del verdadero potencial individual y se enmarcan en la psicología del funcionamiento positivo. Blanco y Díaz (2005), afirman que el bienestar subjetivo viene a ser el resultado de un "balance global" que la persona hace de sus oportunidades vitales, del curso de acontecimientos a los que enfrenta, y de la experiencia emocional derivada de ello: "la Satisfacción con la Vida es el grado en que la persona evalúa la calidad global de su vida en conjunto de forma positiva". Este aspecto sería fundamental en las personas que ya han avanzado por diferentes etapas de su vida, de ahí el interés del estudio en centrarse en un segmento específico de la población y determinar los factores que influyen en la evaluación que las personas mayores de 60 años hacen de su vida, precisando las variables que intervienen en la percepción de su Satisfacción Vital, desde la perspectiva de las variables sociodemográfica, siendo este el primer objetivo de este estudio.

En esta valoración definida como la Satisfacción Vital se encuentra, según Diener (1984), una literatura extensa e incluye estudios que han definido el concepto como felicidad, afecto positivo y bienestar subjetivo. Cabañero, Richart, Cabrero, Orts, Reig, y Tosal (2004) señalan que la Satisfacción Vital es un constructo controvertido en sí mismo, esto probablemente por la baja definición de la variable y la determinación de cuales serían sus componentes. Entre las definiciones encontradas se puede mencionar a Horley (1984), quien afirma que es la "evaluación personal que cada uno hace de sus condiciones particulares, comparada con un referente externo o sus propias aspiraciones”. A su vez George (1981), señala que es una medición cognitiva del ajuste entre los objetivos deseados y los actuales resultados de vida. Apoyando esta visión, Díaz y Sánchez (2001) señalan que la Satisfacción con la Vida es considerada como el componente cognitivo del bienestar subjetivo. Veenhoven (1994; citado en Blanco y Díaz, 2005), evidencia que la Satisfacción con la Vida es el grado en que una persona evalúa la calidad global de su vida en conjunto de forma posi- 
tiva. Esto es en otras palabras, cuanto le gusta a una persona la vida que lleva.

Durán, Extremera, Montalban y Rey (2005), mencionan que la Satisfacción Vital es la evaluación global que la persona hace de su vida, incluyendo el examen de los aspectos tangibles obtenidos, el equilibrio relativo entre aspectos positivos y negativos, comparaciones con un criterio elegido por ella y elaboración de un juicio cognitivo sobre su nivel de satisfacción. De acuerdo a Castellón, Gómez y Martos (2004), la Satisfacción Vital conlleva una valoración subjetiva y la manifestación verbal de esa valoración. Los autores plantean un aspecto básico en el plano de la investigación de la Satisfacción Vital, acerca de la posibilidad de medición, señalan que la única alternativa es preguntarlo directamente a la persona a evaluar. Esto principalmente porque es la propia persona que se encuentra en conocimiento de su situación, de ahí su capacidad de valoración. Díaz y Sánchez (2002) señalan que la satisfacción es entendida como una representación cognitiva resultado de cómo la persona interpreta la satisfacción en diferentes dominios de su vida. Shin y Jonson (1978; citado en Zhang y Leung, 2002), mencionan que la Satisfacción Vital en general ha sido definida por la persona en una evaluación global de su calidad de vida basada en sus propios estándares. En esta dirección Atienza, Pons, Balaguer y García-Merita (2000), señalan que la Satisfacción Vital es una evaluación que refleja una perspectiva a largo plazo. Así, la investigación desde la psicología de la felicidad o bienestar subjetivo se ha incrementado notablemente en las últimas décadas (Alarcón, 2006), sin embargo, los avances en la investigación de la felicidad sugieren que las pruebas para evaluarla son relativamente escasas. En este sentido surge un segundo objetivo en este estudio, que es establecer si la prueba desarrollada por Diener et. al. (1985), SWLS, presenta una validez necesaria para ser aplicada a una muestra local, para adultos mayores.

Para los efectos de esta investigación se utilizará la definición señalada por Seligman y Csikszentmihalyi (2000), que han denominado al Bienestar Subjetivo como una "amplia categoría de fenómenos que incluyen: las respuestas emocionales, la satisfacción en diferentes dominios o áreas y un juicio global de la satisfacción vital”. Frente a este bienestar subjetivo, Veenhoven (1984) y Cole, Peeke y Dolezal (1999) señalan que consta de dos elementos: la cognición, que es definido como la evaluación global que la persona hace de su vida, un índice de ajuste global y el componente emocional, que es mucho más lábil, momentáneo y cambiante constituido por dos dimensiones relativamente independientes; afecto positivo y negativo. Wilson (1967) evidencia que los primeros trabajos que intentaron identificar las variables que influían en estos constructos provienen de una perspectiva sociológica, tratando de definir las variables demográficas que influyen en el bienestar (dinero, edad, sexo, salud o estado civil). En una segunda etapa del estudio de las variables influyentes en el bienestar, se abre una perspectiva psicológica, que Costa y McCrae (1998), la definen como la búsqueda entre la Satisfacción Vital y las características estables de personalidad. Little (1999), toma esta segunda perspectiva par incluir en 
el análisis de variables las metas personales y proyecciones a futuro, denominando una perspectiva cognitivo-motivacional, basado en el sustento de que las metas personales se relacionan directamente con el bienestar psicológico. Frente a estas dos posturas de estudio del bienestar, Castro y Díaz (2002) afirman que actualmente se trabaja en una línea de integración de variables, considerando las características estables de personalidad, metas u objetivos y el contexto de la persona, como influyentes en la Satisfacción Vital de las personas.

Estas tres perspectivas son el fruto de la búsqueda del por qué las personas están satisfechas con la vida. Ello ha conducido a un análisis de los determinantes del bienestar, intentado averiguar qué es causa y qué es efecto (Díaz y Sánchez, 2002).

\section{Causas y Consecuencias}

Frente a las variables o factores determinantes de la Satisfacción Vital, Díaz y Sánchez (2002) señalan que no aparecen relaciones causaefecto claras, sino más bien modelos que dan cuenta de relaciones bidireccionales y circulares entre la satisfacción con la vida en general y la satisfacción con diferentes áreas vitales. Castro y Sánchez (2000) afirman que no es posible establecer fehacientemente qué es causa y qué es consecuencia del bienestar, las investigaciones en el terreno de la satisfacción, en su mayor parte, han recabado datos transversalmente y han establecidos las correlaciones entre ellos. Frente a esto los autores explican la existencia de dos teorías contrapuestas: la primera denominada Botton Up que consiste en que las variables frecuentemente tratadas en los estudios sobre la satisfacción (acontecimientos del ciclo vital, áreas vitales, estándares de referencia, expectativas, aspiraciones, etc.) son las responsables de la satisfacción de la vida como un todo. Así la satisfacción se entiende como una sumatoria de estas variables, siendo el resultado de haber tenido muchos momentos felices en la vida. Frente a esto se puede señalar que los aspectos fundamentales de esta teoría pasan por sucesos y aspectos contextuales que aportan en los niveles de la Satisfacción Vital de las personas. Se señala una segunda teoría que intenta explicar el fenómeno, denominada Top Down, completamente opuesta a la anterior, ya que supone que el bienestar general de las personas es el responsable de las otras variables comentadas. El bienestar es una disposición general de la personalidad y tiene una característica estable en la vida de las personas. Esto implica que existen personas "felices" y que este sentir lo trasladan a sus diferentes esferas de vida y de esto surge la satisfacción en su quehacer. Para el desarrollo del conjunto de este estudio, tiene como sustento a la primera teoría, ya que pretende estudiar y medir cómo diferentes variables de contexto aportan en los índices de satisfacción vital a través del ciclo vital, de esta forma en particular se exploran cómo variables sociodemográficas, explican y logran diferenciar la satisfacción vital de personas mayores de 60 años.

En relación a la Satisfacción Vital a través del tiempo de las perso- 
nas, Chamberlain y Zika (1992), comentan que si bien hay numerosos estudios que afirman la estabilidad a largo plazo de la satisfacción, muchos no toman en cuenta que pueda cambiar de forma predecible para algunas personas. En este sentido Headey y Wearing (1989), explican que si bien la satisfacción es estable, es sensible a las circunstancias cambiantes. De esto se puede entender que a través del ciclo vital las variables moderadoras de la Satisfacción Vital tenderán a ir modificándose en tanto se va alcanzando ciertas etapas evolutivas. En este sentido Ericson (1996), señala que el bienestar subjetivo se encuentra relacionado con el contacto social y las relaciones interpersonales lo que avalaría por tanto la importancia de personas significativas en los niveles de Satisfacción Vital, que claramente las personas van cambiando conforme se avanza en el ciclo vital.

\section{Factores Sociodemográficos y Satisfacción Vital}

Uno de los principales intereses de este estudio se centra en discriminar cuáles de las variables sociodemográficas tales como: enfermedades crónicas, importancia de la religión en la vida, ingreso total familiar, situación de vivienda y situación de hogar, pueden explicar de mejor forma el incremento de la Satisfacción Vital en una muestra de adultos mayores de 60 años. Por otro lado, dentro de la literatura científica se ha encontrado que las variables demográficas para predecir la Satisfacción Vital incluyen la edad, sexo, ingresos, raza, ocupación (empleo), educación, estatus socio-económico, religión y matrimonio (Zhang, 2005). Éstas son de fácil medición y sus errores son relativamente pequeños. A estas variables hay que sumar aquellos aspectos relacionados con la influencia social que existe con los índices de Satisfacción Vital. Ranzijn (2002), señala que existe un creciente reconocimiento de que la salud psicológica, y hasta en cierta medida la salud física, depende al menos en algún grado de sentirse integrado a una comunidad. Esto afirma el sentido de que el apoyo social es una de las fuentes importantes en la predicción de la Satisfacción Vital, la felicidad y las relaciones afectivas cercanas. La necesidad de pertenencia y de relacionarse socialmente está dada por el carácter de protección y de reconocimiento que necesita el ser humano (Cuadra y Florenzano, 2003). Para Fernández y García (1994), el concepto de apoyo social tiene que ver con algo tan cotidiano y tan inmediato como las relaciones sociales que cada uno establece en su entorno, que suponen una fuente de experiencias de muy diverso tipo entre las cuales son especialmente importantes las que se refieren a la recepción de ayuda en sus formas materiales, emocionales o de cualquier otro tipo. De esto se desprende el tercer objetivo de este estudio, en que se espera que los niveles de satisfacción vital entre las parejas no evidenciarán diferencias significativas entre ellos, es decir, las parejas tenderán a presentar niveles similares de satisfacción vital, esto implica señalar que la presencia de una persona significativa tendría un mayor "peso" en los niveles de Satisfacción Vital. Brown, Mounts, Lamborn y Steinberg (1993), señalan que mantener relaciones positivas con amigos y amigas, en las que existe intimidad, seguridad y apoyo influye de forma muy significativa en la autoestima y Satisfacción Vital. Por su parte, Fernández y García 
(1994) afirman que el apoyo social tiene un efecto mediador o amortiguador frente a los estresores del individuo, esto porque presenta un efecto beneficioso sobre la calidad de vida de los sujetos.

En relación a las variables sociodemográficas, Bonavía y Quintanilla (1998), encuentran que las variables situacionales como el nivel de ingresos y el estatus (definido como el nivel jerárquico dentro de una organización) no presentan influencia en la Satisfacción Vital de las personas, por lo que los aspectos de apoyo y cercanía social tienden a tener una mayor preponderancia al momento de establecer los niveles de Satisfacción Vital. En contraparte a lo relacionado con los ingresos económicos, Richards (1989) concluye en su estudio que las mujeres se sienten más estigmatizadas por ser pobres más que por ser madres solteras, por lo que se puede señalar que la influencia de los factores económicos no es posible aislarla, ya que se deberían considerar otras variables sociodemográficas. En este sentido la evidencia empírica sobre la influencia del ingreso económico es muy heterogénea, encontrándose resultados a favor y en contra de la relación con la felicidad y la Satisfacción Vital (Peiró, 2001). Para la realidad local en el cual se centra este estudio, Lee (2008) ha encontrado que el nivel de ingresos económicos correlaciona positiva y significativamente con la variable Satisfacción Vital, sin embargo se presenta como una relación muy débil $(0,210)$ pero no alejada de la encontrada por Argyle (1987) cuya relación entre la satisfacción en relación a los ingresos económicos en distintos países correlaciona entre 0,15 a 0,25, lo que hace pensar que los ingresos económicos aportan en cierta medida a la satisfacción vital, sin embargo no es determinante de ésta (Lee, 2008). Por otro lado, frente a la salud de las personas, Castro y Sánchez (2000) señalan que a una mayor experiencia de salud física y psicológica existe una mayor percepción de satisfacción global con la vida. Moyano y Ramos (2007), en su estudio identifican dos variables que influyen en la Satisfacción Vital, señalando que las mujeres están más satisfechas que los hombres, y que las personas casadas obtienen puntajes en promedio significativamente superiores que las personas solteras. De esto se desprende que las variables sexo y estado civil, tienen una influencia en la medición de la Satisfacción Vital.

\section{Satisfacción Vital y Adultos Mayores}

La Satisfacción Vital ha sido un gran punto de interés en la investigación gerontológico-social por más de 40 años (Inga y Vara, 2006). En los últimos años se ha generado un especial interés por la investigación relacionada con la tercera edad (Mella et al., 2004). Este creciente interés se puede deber al considerable aumento de personas que viven esta realidad, el cual hace surgir el interés a escala mundial por sus condiciones de vida y las necesidades específicas de este grupo, cobrando relevancia en los últimos años, las investigaciones relacionadas con el bienestar subjetivo del adulto mayor y de las variables específicas que tienen una influencia en él. Por lo que es una realidad que las sociedades occidentales están envejeciendo, aunque buena parte de las personas mayores pueden desenvolverse 
adecuadamente en el día a día, los porcentajes de mayores dependientes van en aumento (López y Crespo, 2007). Frente a lo anterior, estudios realizados por el Instituto Nacional de Estadísticas (2000), evidencian que se espera que para el año 2010 la población en Chile que va a superar los 60 años, corresponderá a un 12,94\% de la población total. Y aunque el envejecimiento se presenta hoy como una tendencia universal, su intensidad varía, sobre todo entre las distintas regiones (Barrón, Mardones, Vera, Ojeda y Rodríguez, 2006). Actualmente, los países desarrollados presentan un número elevado de personas mayores y esta tendencia va constantemente en aumento. Para García (2007), el aumento de la esperanza de vida con el consiguiente envejecimiento de la población mundial es un logro. El problema es que ni la sociedad ni la economía están adaptadas para ese cambio que plantea nuevos problemas de atención sanitaria, recursos económicos, empleo, educación, entre otros. Este envejecimiento demográfico de nuestra población constituye el gran desafío del tercer milenio, pues trae consigo transformaciones notables en todas las esferas de la vida familiar, social, sanitaria, económica, política y educacional. Pereira (1999), señala que el envejecimiento poblacional conlleva una creciente demanda de servicios y atenciones que son propios de las necesidades que generan los adultos mayores, las que se enmarcan en áreas diversas como la economía, vivienda y urbanismo, salud, participación social y educación. En relación al proceso de envejecimiento de la población, Castro et al. (2007) sugieren que se torna necesario no sólo informar sobre la calidad de vida de la tercera edad, sino que también establecer programas colectivos de atención completa e interdisciplinaria que atenúen varios aspectos del proceso de envejecimiento. Los procesos de envejecimiento y el cúmulo de pérdidas psicosociales que acontecen durante la vejez parecen determinar en algunos ancianos su incapacidad para percibir sus competencias y habilidades $\mathrm{y}$, desde luego, los aspectos positivos del entorno que les rodea y de la vida en general (Mella et. al., 2004). Por lo que puede ser valioso definir qué aspectos son los que influyen en la satisfacción vital de las personas de la tercera edad, para que en su posible modificación, pueda influir en la calidad de vida de éstos. Inga y Vara (2006) plantean que para el adulto mayor, la familia sigue siendo la principal fuente de apoyo emocional, de seguridad, cuidado y afecto, ya que constituye un mecanismo natural de atención. En relación al apoyo social percibido para este grupo etario, FernándezBallesteros et al. (1992), señalan que las relaciones positivas y el poseer redes de apoyo, influyen en la mantención de una autoimagen positiva y una autoestima elevada, aspectos centrales del bienestar subjetivo. Del mismo modo Mella et. al. (2004), confirman que el bienestar subjetivo está determinado principalmente por el grado de apoyo que el adulto mayor siente que tiene por parte de su entorno. Castellón et. al (2004), señalan que para el adulto mayor las variables psicosociales, según la mayoría de los estudios que parecen predecir mejor la satisfacción vital son; la salud, las habilidades funcionales, los contactos sociales y la actividad de la persona. Argyle (1992), en relación a los determinantes de la felicidad afirma que la salud es importante en todas las edades, pero especialmente para el adulto mayor, para quienes suele ser un problema y una fuente de preocupación. Los ingresos son relativamente poco importantes para 
los jóvenes y muy importante para personas mayores, puesto que también en este caso tienen menos.

\section{Método}

\section{Objetivos}

1. Validar para una muestra local de adultos mayores el instrumento de SWLS (Diener et. al., 1985), en su versión al castellano.

2. Determinar la importancia e influencia de las variables sociodemográficas en los índices de Satisfacción Vital en adultos mayores.

3. Determinar si existen o no diferencias significativas de puntuaciones medias en el instrumento de SWLS (Diener et. al., 1985), entre parejas, en los índices de Satisfacción Vital de adultos mayores.

\section{Muestra}

122 personas mayores de 60 años de la ciudad de Arica. Las edades fluctuaron entre los 60 y los 82 años, con edad media de 66 años. La muestra estaba constituida por $52,5 \%$ de hombres y $47,5 \%$ de mujeres. Se entrevistaron a 36 parejas para efectuar las comparaciones de media en la medición de la Satisfacción Vital.

\section{Instrumentos}

Cuestionario de Satisfacción Vital (SWLS): Diener et. al. (1985), SWLS. Para esta investigación se consideró la traducción del cuestionario original ofrecida por Seligman (2003), manteniendo inalterable la versión original de 5 reactivos, con 7 posibilidades de respuesta que van desde (1) Fuertemente en desacuerdo a (7) Fuertemente de acuerdo. Este instrumento es una medida global acerca del grado percibido de satisfacción con la vida y está formado por los siguientes ítems: "En la mayoría de los aspectos, mi vida se acerca a mi ideal”, "Las condiciones de mi vida son excelentes", "Estoy completamente satisfecho con mi vida"; "Hasta ahora, he conseguido las cosas que para mí son importantes en la vida” y "Si pudiera vivir de nuevo, no cambiaría nada”. En mediciones pasadas los rangos de confiabilidad de alfa de cronbach oscilan entre .89 y .79 (Pavot y Diener, 1993). El análisis factorial del instrumento original realizado por Diener et. al. (1985) arrojó como resultado un instrumento unifactorial que explica el $66 \%$ de la varianza de la escala, mediante los ejes principales. El instrumento es ampliamente utilizado para diferentes muestras, presentando características de unifactorial. Cabañero, et. al. (2004), validaron el instrumento con mujeres embarazadas y puérperas obteniendo resultados similares a los originales con un _.82, mientras que Palomera y Brackett (2006) obtuvieron un _.86 y Extremera, Durán y Rey (2005) un _.85. Existen investigaciones realizadas con la traducción del instrumento al español que 
han reducido de 7 a 5 posibilidades de respuesta (Atienza, et. al. 2000) obteniendo _.84, en el desarrollo de su investigación, los autores identifican entre diferentes estudios a 33 investigaciones que han ocupado el cuestionario en su versión original en inglés y 4 estudios de tipo transcultural. Por lo que la utilización y aceptación del instrumento es ampliamente reconocida.

Cuestionario de variables sociodemográficas: desarrollado para esta investigación, incluyó las siguientes variables: Enfermedad Crónica, variable dicotómica que explora la presencia o no de enfermedades de carácter crónico que los encuestados pueden presentar. Gfellner (1989, citado en Inga y Vara, 2006), señala que las percepciones de salud son un indicador de la satisfacción de vida en comparación con el número de enfermedades crónicas. Según lo planteado por Gerstorf, Lövdén, Röcke, Smith y Lindenberger (2007), los problemas de salud tienen una relación negativa con el bienestar subjetivo, en términos de las medidas medias y cambio en el tiempo. Argyle (1992), señala que la salud no sólo influye en la felicidad y la satisfacción, sino que la relación es especialmente importante en el caso de los ancianos. Importancia de la Religión en la vida, escala que evalúa la importancia que tiene para los componentes de la muestra la práctica de una religión determinada. Se consideró la importancia más que el hecho de poseer o no una creencia, la escala va de (1) sin importancia a (4) muy importante. Seligman (2003) señala que la relación causal entre religión y vida más sana y de carácter más social no es ningún misterio. Ingreso Total Familiar; se pretende evidenciar si existen diferencias entre los niveles de ingreso económico con la Satisfacción Vital, para esto se establecen las posibilidades de respuesta en escala, las posibilidades de respuesta van de (1) 120.000-240.000 a (5) más de 1.800 .000 , en pesos chilenos. Argyle (1993), señala que el nivel de ingresos influye de modo claro, aunque escaso en la satisfacción. El conjunto de investigaciones realizadas hasta la fecha, no llega a aclarar la relación que mantienen los indicadores objetivos (entre ellos el nivel de ingresos y el status socio-económico) con la calidad de vida ni la satisfacción vital, abundando los resultados contradictorios (Bonavía y Quintanilla, 1998). Situación de Vivienda, escala de medición en que se señalaba la situación de vivienda de los evaluados, estableciendo las posibilidades en escala, con valores de (1) Allegado a (4) Casa o departamento propio sin deuda. Rohe y Stegman (1994), señalan que los dueños de casa poseen mayor satisfacción que las personas que arriendan casas, y que esta variable es mucho más importante que la edad, ingreso económico y otras variables demográficas. Situación de Hogar, escala de medición que señala con quién viven los integrantes de la muestra, va desde 1) solo a 4) cónyuge e hijos. Se espera que aquellos que viven en compañía con su familia completa puedan presentar niveles más elevados de satisfacción vital, en comparación a quienes viven de forma solitaria. La importancia de la inclusión de estas variables en el cuestionario, son las posibles correlaciones con los niveles de Satisfacción Vital de los integrantes de la muestra. 


\section{Diseño y Procedimiento}

Se utilizó un diseño no-experimental de tipo descriptivo correlacional, ex-post facto. La participación de los integrantes de la muestra fue completamente voluntaria, a cada uno de éstos se les entregaron los dos cuestionarios mencionados. Aquellos que no se encontraban con sus parejas en los momentos de la medición se les consideró de forma individual, en cambio, quienes contaban con sus parejas fueron tabulados en pares, para poder determinar uno de los aspectos principales de esta investigación, que es comprobar la similitud de los niveles de Satisfacción Vital. Para la aplicación de los instrumentos se utilizaron alumnos de psicología de $4^{\circ}$ año, previamente capacitados. Los datos recogidos fueron analizados con el paquete estadístico SPSS 14.0.

\section{Resultados}

En relación al primer objetivo de estudio referido a la validación del instrumento para la muestra local, los resultados para la Escala de Satisfacción con la Vida (Diener, et. al., 1985) señalan que éste presenta una muy buena consistencia interna (_.856), en relación con los 5 ítemes de la prueba.

Al realizar el análisis factorial a la prueba, éste arroja mediante el método de ejes principales, con rotación varimax, una escala unifactorial que explica el $66 \%$ de la varianza de la prueba. Lo destacable es que la carga factorial de cada uno de los ítemes de la escala, se muestran lo suficientemente robustos, evidenciando altos pesos factoriales que oscilan entre .74 y .87 .

Teniendo ya la escala validada es preciso evidenciar los puntajes obtenidos por la muestra evaluada, las puntuaciones medias en la Escala de Satisfacción con la Vida (Diener, et. al., 1985), se observan en la tabla 1.

Tabla 1

\begin{tabular}{|l|c|c|}
\hline \multicolumn{2}{|c|}{ Puntuaciones Medias Escala SWLS y Categoria SWLS } \\
\hline \multicolumn{1}{|c|}{ Muestra } & Puntuaciones Media SWLS & Categoria SWLS \\
\hline Población Total & 28,08 & Ligeramente Satisfecho \\
\hline Hombres & 28,59 & Ligeramente Satisfecho \\
\hline Mujeres & 27,52 & Ligeramente Satisfecho \\
\hline Casado(a) & 28,74 & Ligeramente Satisfecho \\
\hline Viudo(a) & 26,57 & Ligeramente Satisfecho \\
\hline
\end{tabular}

Así, los resultados obtenidos en las mediciones de la Satisfacción con la Vida evidencian a los hombres (28,59 puntos) con mayores niveles de satisfacción que las mujeres (27,52 puntos), sin embargo estas diferen- 
cias no son significativas; lo mismo ocurre de acuerdo a la condición de pareja, las personas casadas muestran mayores niveles de Satisfacción Vital que las personas viudas, pero estas diferencias tampoco llegan a ser significativas. Es interesante destacar que la medición de la Satisfacción con la Vida, clasifica a la población total (ya sean hombres, mujeres, viudos o casados) como ligeramente satisfechos.

Por otro lado, en la tabla 2 se evidencia para el grupo de muestra total la existencia de correlaciones de carácter positivo y estadísticamente significativo de las variables sociodemográficas (Enfermedad Crónica, Ingreso Total Familiar, Situación de Vivienda y Situación de Hogar) y las puntuaciones medias de la Escala de Satisfacción con la Vida (Diener, et. al., 1985). Sin embargo, unas variables presentan mayor fuerza en la relación que otras. Por lo que se podría decir que la Satisfacción con la Vida se relaciona más fuertemente con la Variable Situación de Vivienda, seguida por Ingreso Total Familiar, Situación de Hogar y finalmente con Enfermedad Crónica. Lo que podría sugerir que el poseer una vivienda propia se relaciona más fuertemente con la Satisfacción Vital que la ausencia de una enfermedad crónica (tabla 2).

\section{Tabla 2}

\begin{tabular}{|c|c|c|}
\hline \multicolumn{3}{|c|}{ Análisis de correlaciones de variables sociodemográficas con escala SWLS } \\
\hline Grupo & Variable & Puntuación total SWLS \\
\hline \multirow[t]{4}{*}{ Muestra Total } & Enfermedad Crónica &, $218\left({ }^{\star \star}\right)$ \\
\hline & Ingreso Total Familiar &, $307\left({ }^{\star \star}\right)$ \\
\hline & Situación de Vivienda &, $313\left({ }^{* *}\right)$ \\
\hline & Situación de Hogar & ,227(*) \\
\hline Grupo Hombres & Ingreso Total Familiar & ,293(*) \\
\hline \multirow[t]{4}{*}{ Grupo Mujeres } & Actividad Laboral & ,323(*) \\
\hline & Enfermedad Crónica & ,278( $\left(^{\star}\right)$ \\
\hline & Ingreso Total Familiar & ,286(*) \\
\hline & Situación de Hogar & ,437(*) \\
\hline
\end{tabular}

**Correlation is significant at the 0.01 level (2-tailed).

*Correlation is significant at the 0.05 level (2-tailed).

Ahora bien, con respecto a la variable sexo (grupo hombres y grupo mujeres), los análisis arrojan sólo una correlación positiva y significativa en los hombres entre el Ingreso Total Familiar y la Satisfacción con la Vida (,293). Y, a pesar de que la fuerza de esta relación es más débil que la del Ingreso Total Familiar para la muestra total $(, 307)$ es la más importante para los hombres de la muestra, sugiriendo la confirmación del rol que asumen generalmente como "proveedores” dentro de una familia. 
Por otro lado, se observa que para las mujeres en comparación con los hombres, aumenta el número de variables sociodemográficas que correlacionan de forma positiva con la Satisfacción Vital. Llama la atención que para este grupo correlaciona una nueva variable con SWLS, que es el desarrollo de una Actividad Laboral, sugiriendo que aquellas mujeres que si se encuentran empleadas y percibiendo ingresos tienden a demostrar mayores índices de Satisfacción Vital. Del mismo modo es más importante para las mujeres de la muestra, el vivir dentro de un núcleo familiar que esté compuesto por parejas y/o hijos.

Para un análisis más detallado es preciso realizar un análisis de regresión de orden ordinal por las características de las variables. Para la muestra total, los valores de Nagelkerke evidenciaron un valor de Pseudos R-Cuadrado de ,260 con significancia de ,000. Esto con las variables de Enfermedad Crónica, Ingreso Total Familiar, Situación de Vivienda y Situación Familiar.

$\mathrm{Al}$ comparar a las personas que reportan alguna enfermedad de tipo crónica frente a quienes no la padecen, las variables sociodemográficas que presentan correlaciones con la Satisfacción Vital se establecen en la tabla 3.

\section{Tabla 3}

\begin{tabular}{|c|c|c|c|}
\hline \multicolumn{4}{|c|}{$\begin{array}{l}\text { Análisis de correlaciones de variables sociodemográficas con escala SWLS } \\
\text { CON ENFERMEDAD CRÓNICA - SIN ENFERMEDAD CRÓNICA }\end{array}$} \\
\hline Variable & $\begin{array}{c}\text { Puntuación total SWLS } \\
\text { Enfermedad Crónica }\end{array}$ & $\begin{array}{l}\text { Puntuación total SWLS } \\
\text { Sin Enfermedad Crónica }\end{array}$ & $\begin{array}{l}\text { Diferencias de } \\
\text { Correlaciones }\end{array}$ \\
\hline Hijos &, $247\left(^{\star}\right)$ &,- 076 & 0,04 \\
\hline Situación Hogar &, $358\left({ }^{\star \star}\right)$ &,- 128 & 0,00 \\
\hline Ingreso total familiar &, $340\left({ }^{* *}\right)$ & ,092 & 1 \\
\hline Situación de Vivienda &, $259\left({ }^{*}\right)$ &, $343\left({ }^{*}\right)$ & 0,68 \\
\hline
\end{tabular}

**Correlation is significant at the 0.01 level (2-tailed).

*Correlation is significant at the 0.05 level (2-tailed).

Se pueden dividir en dos las variables sociodemográficas que correlacionan con la Satisfacción Vital por parte de los integrantes de la muestra que presentan enfermedad crónica y quienes no presentan una enfermedad crónica. Para quienes si presentan una enfermedad crónica, sus niveles de Satisfacción Vital correlacionan con variables de índole más "social” como lo es el tener hijos y vivir en compañía (variables Hijos y Situación Hogar). De esto se puede señalar que, tener hijos y vivir en compañía para quienes presentan enfermedades crónicas se constituye en variables que ayudarían a los integrantes de la muestra a presentar mayores índices de Satisfacción Vital, existiendo diferencias significativas en las correla- 
ciones de estas variables, entre quienes presentan una enfermedad crónica y quienes no la presentan.

En cambio para quienes no presentan una enfermedad crónica, sus niveles de Satisfacción Vital se asocian con variables de orden más "material” como lo es el ingreso económico a nivel familiar y la posesión de una vivienda (variables Ingreso Total Familiar y Situación de Vivienda), no estableciéndose diferencias significativas entre correlaciones entre quienes sufren de una. Por lo que se puede decir que la diferencia de la Satisfacción Vital entre quienes padecen de alguna enfermedad crónica no pasa por factores de orden material sino por un factor social, que es este caso es el de sentirse acompañados por la presencia de figuras importantes.

Uno de los principales objetivos de esta investigación era determinar si existían diferencias significativas en las puntuaciones medias entre parejas. Concordando con Argyle (1992), quién señala que en el matrimonio existe una reciprocidad que es importante en referencia a la satisfacción vital. De acuerdo a los resultados no se evidencian diferencias de medias significativas en las puntuaciones medias en SWLS, por parte de las parejas mayores de 60 años de la muestra. Con este resultado se comprueba el tercer objetivo de la investigación, es decir, se establece por tanto que quienes mantienen una relación de pareja, mantendrán puntuaciones similares en la Satisfacción Vital. Estos resultados pueden ser concordantes por lo planteado por Seligman (2003), quién señala que el matrimonio por si solo es un poderoso factor para presentar altos niveles de felicidad.

\section{Conclusiones}

Los resultados del estudio indican que la versión en castellano de la Escala de Satisfacción con la Vida (Diener et al., 1985), posee adecuadas propiedades psicométricas para ser aplicado a la población local. Por lo que entrega una medida en castellano del constructo de satisfacción con la vida, considerado como un juicio global en el que el sujeto utiliza sus propios criterios para sopesar los distintos aspectos de su vida (Diener et al., 1985). El análisis factorial demostró que la escala se comporta como una estructura unifactorial, explicando un adecuado porcentaje de la varianza total (66\%), con igual porcentaje de varianza explicada al obtenido por Diener et al. (1985). Por otra parte, los pesos factoriales de los ítems se encuentran dentro del rango obtenido por investigaciones anteriores. Además la escala tiene una muy buena consistencia interna, comparable con los rangos de investigaciones anteriores y la obtenida por el original, es importante consignar que no existe ningún ítem cuya eliminación aumente la consistencia de la prueba. Con esto se cumple el primer objetivo de esta investigación que es el validar el instrumento desarrollado por Diener et al. (1985) para una muestra local.

En términos generales los integrantes de la muestra presentan elevados niveles de Satisfacción Vital, evidenciando diferencias en las puntua- 
ciones medias entre hombres y mujeres, sin embargo estas diferencias no son estadísticamente significativas. Estos resultados son concordantes por lo propuesto por Argyle (1992), quien señala que hay pocas diferencias entre los sexos respecto a la satisfacción vital general o a las emociones positivas.

Las variables sociodemográficas seleccionadas en este estudio (enfermedades crónicas, importancia de la religión en la vida, ingreso total familiar, situación de vivienda y situación de hogar) llegan a explicar un porcentaje aceptable para el componente de la Satisfacción Vital, considerando que es el juicio global que la persona realiza de los diferentes aspectos de su vida.

Se observan diferencias en las correlaciones de las variables sociodemográficas. Para los hombres sólo la variable de "ingresos económicos” presenta un relación con la Satisfacción Vital, en cambio para las mujeres las variables aumentan, incorporando variables relacionadas con la compañía y cercanía de su familia. Frente a esto Stearns y Stearns (1986, citado en Alcalá, Camacho, Giner e Ibáñez, 2006), señalan que el rol de la mujer implica el cuidado de los miembros de su familia, dentro de la esfera privada del hogar, que requiere sensibilidad emocional. Por el contrario, el papel del hombre implica ganar un salario para su familia en el ámbito público del trabajo, que requiere la reserva emocional. Esto lo señalan según las teorías sociales que han sido esgrimidas para entender las diferencias expresivas de la emoción según el género. De esto se puede señalar que la concepción de la emoción en sí afectan en las variables a considerar para la Satisfacción Vital, según la variable sexo.

Como las variables sociodemográficas señaladas, tienen un rol importante en la explicación de la Satisfacción Vital, se puede entender que no existan diferencias significativas en las mediciones de la escala SWLS entre las parejas de la muestra. Esto implicaría que las parejas tenderían a “equilibrarse” en los índices de Satisfacción Vital, por lo que el tercer objetivo de la investigación se cumple. Las variables sociodemográficas consideradas para la explicación de esta investigación, explican un porcentaje aceptable y dentro de investigaciones anteriores, por lo que éstas se convierten en un buen predictor de la satisfacción vital en adultos mayores para una muestra local.

Se evidenciaron diferencias entre los adultos mayores que padecen de enfermedades crónicas y quienes no en la valoración que se hace al contar con apoyo social, ya sea por la presencia de hijos o por vivir en compañía. Esto refleja que el apoyo social y familiar es importante en los índices de satisfacción vital por parte de adultos mayores.

\section{Limitaciones y Proyecciones}

A pesar de que la muestra evaluada presenta altos niveles de satis- 
facción vital, se puede establecer como una limitación de las características de la muestra el no encontrar diferencias significativas entre las personas que tienen pareja y aquellos viudos o solteros, debido también a que el número de las personas solas era significativamente menor al número de personas que contaban con pareja. En la misma dirección se establece una segunda limitación del estudio que se presenta al verificar las diferencias existentes entre las personas con una alta valoración e importancia de la creencia religiosa en su vida frente a quienes no le asignan una gran importancia, ya que tampoco se encontraron diferencias significativas entre ambos grupos. Esto se puede deber principalmente a que las personas que no asignaban una alta importancia de la creencia religiosa en sus vidas representan sólo el 2,5\% de la muestra total. En este sentido, se puede destacar a Seligman (2003) quien señala que no existe una relación clara entre religión y un mayor grado de felicidad.

Finalmente, es interesante plantear para futuras investigaciones el poder establecer una cadena causal de la Satisfacción Vital dentro de la familia por generaciones, ampliar este estudio a diferentes contextos sociales como asilos de ancianos, hospitales y organizaciones laborales que cuenten con empleados mayores de 60 años, e incluir variables que puedan establecer medidas de contacto social como la participación de deportes, centros comunitarios, etc. 


\section{Bibliografía}

Alarcón, R. (2006), “Desarrollo de una escala factorial para medir la felicidad”, Revista Interamericana de Psicología, Vol. 40, n 1, pp. 99-106.

Argyle, M. (1993), La psicología de la felicidad, Alianza editorial S.A., Madrid.

Atienza, F., Pons, D., Balaguer, I. y García_Merita, M. (2000), "Propiedades psicométricas de la escala de satisfacción con la vida en adolescentes”. Psicothema. Vol. 12, $\mathrm{n}^{\circ}$ 2, pp. 314-319.

Ballesteros, B., Medina, A. y Caicedo, C. (2006), “El bienestar psicológico definido por asistentes a un servicio de consulta psicológica en Bogotá Colombia”, Univ. Psychol. Bogotá. Vol. 5, n 2, pp. 239-258.

Barrón, M., Mardones, M., Vera, S., Ojeda, G. y Rodríguez, F. (2006), “Comparación de estilos de vida de la población económicamente activa mayor de 65 años entre las ciudades de Chillan y Valparaíso, Chile”. Teoría, Vol. 15, nº 1, pp. 33-44.

Bonavía, T. y Quintanilla, I. (1998), "El efecto de los ingresos, el nivel jerárquico, la cultura empresarial y las creencias de los directivos sobre su satisfacción vital”, Revista de Psicología del Trabajo y de las Organizaciones. Vol. 14, n 2, pp. 217-231.

Brown, B., Mounts, N., Lamborn S. y Steinberh, L. (1993), "Parenting practices and peer group affiliation in adolescence”, Child Development. Vol. 64, pp. 467-482.

Cabañero, M., Richart, M., Cabrero, J., Orts, M., Reig, A. y Tosal, B. (2004), "Fiabilidad y validez de la Escala de Satisfacción con la Vida de Diener en una muestra de mujeres embarazadas y puérperas”, Psicothema. Vol. 16, $\mathrm{n}^{\circ} 3$, pp. 448-455.

Castellón, A., Gómez, M. y Martos, A. (2004), “Análisis de la satisfacción en los mayores de la Universidad de Granada”, Rev. Mult. Gerontol. Vol. $14, \mathrm{n}^{\circ} 5$, pp. 252-257.

Castro, A. y Díaz, J. (2002), “Objetivos de vida y satisfacción vital en adolescentes españoles y argentinos”, Psicothema. Vol. 14, n 1, pp. 112117.

Castro, A. y Sánchez, M. (2000), “Objetivos de vida y satisfacción autopercibida en estudiantes universitarios”, Psicothema. Vol. 12, $\mathrm{n}^{\circ} 1$, pp. 87-92.

Castro, P., Tahara, N., Rebelatto, J., Driusso, P., Aveiro, M. y Oishi, J. (2007), "Influência da universidade aberta da terceira idade (uati) e do 
programa de revitalização (revt) sobre a qualidade de vida de adultos de meia-idade e idosos”, Revista brasileira de fisioterapia, Sao Carlos. Vol. 2, no 6, pp. 461-467.

Cole, D. A., Peeke, L. y Dolezal, S. (1999), “A longitudinal study of negative affect and self-perceived competence in young adolescents”, Journal of Personality and Social Psychology. Vol. 77, n 4, pp. 851-862.

Cornejo, M. y Lucero, M. (2005), “Preocupaciones vitales en estudiantes universitarios relacionados con bienestar psicológico y modalidades de afrontamiento”, Fundamentos en Humanidades. Año VI, no 2 (12/2005), pp. 143-153.

Costa, P. y McCrae, R. (1998), “Estados de ánimo y personalidad en la etapa adulta”. En Sánchez- López y Quiroga Estévez (Eds). Perspectivas Actuales en la Investigación Psicológica de las Diferencias Individuales (pp. 295-310), Centro de Estudios Ramón Areces SA, Madrid.

Cuadra, H. y Florenzano, R. (2003), “El bienestar subjetivo: Hacia una psicología positiva”, Revista de psicología de la Universidad de Chile. Vol. 12 n $^{\circ}$, pp. 83-96.

Chamberlain, K y Zika, S. (1992), "Stability in subjective well being over short time periods”, Social indicators research. Vol. 26, pp. 101-117.

Dávila, M. y Díaz, J. (2005), “Voluntariado y satisfacción vital”, Intervención psicosocial. Vol. 14 n 1, pp. 81-94.

Díaz, J. y Sánchez, M. (2002), “Relaciones entre estilos de personalidad y satisfacción autopercibida en diferentes áreas vitales”, Psicothema. Vol. $14 \mathrm{n}^{0} 1$. pp. 100-105.

Díaz, J. y Sánchez, M. (2001), “Relevancia de los estilos de personalidad y las metas personales en la predicción de la satisfacción vital”, Anales de Psicología. Vol. 17, nº 2, pp. 151-158.

Diener, E., Suh, E., Lucas, R., y Smith, H. (1999), “Subjective well-being: Three decades of progress”, Psychological Bulletin. Vol. 125, $\mathrm{n}^{\circ}$ 2, pp. 276-302.

Diener, E., Emmons, R., Larsen, R. y Griffin, S. (1985), “The satisfaction with life scale. Journal of Personality Assessment”, 49, 71-75. Citado en Seligman, M. La Auténtica Felicidad. (2003), Ediciones B, Barcelona.

Diener, E. (1984), “Subjective well-being”. Psychological Bulletin. Vol. 95, pp. 542-575.

Durán, M., Extremera, N., Montalbán, F. y Rey, L. (2005), “Engagement y burnout en el ámbito docente: Análisis de sus relaciones con la satisfac- 
ción laboral y vital en una muestra de profesores”, Revista de Psicología del Trabajo y de las Organizaciones. Vol. 21, n 12, pp. 145-158.

Ericson, R. (1996), “Descripciones de la igualdad: el enfoque sueco de la investigación sobre el bienestar”. En Nussbaum, M. y Sen, A. (comps.): La calidad de vida (pp.101-120). F.C.E. México.

Extremera, N., Durán, A. y Rey, L. (2005), “La inteligencia emocional percibida y su influencia sobre la satisfacción vital, la felicidad subjetiva y el engagement en trabajadores de centros para personas con discapacidad intelectual”. Ansiedad y estrés. Vol. 11 nº 1, pp. 181-184.

Fernández-Ballesteros, R., Izal, M., Montorio, I., González, J. y Díaz, P. (1992), Evaluación e intervención psicológica en la vejez. Ed. Martínez Roca, Barcelona.

Fernández J. y García, A. (1994), “Redes de Apoyo Social en usuarios del servicio de ayuda a domicilio de la tercera edad”, Psicothema. Vol. $6 \mathrm{n}^{\circ} 1$, pp. 39-47.

García, N. (2007), "La educación con personas mayores en una sociedad que envejece”, Horizontes educacionales. Vol. 12, n 2, pp. 51-62.

George, I. (1981), “Subjetive well-being. Conceptual and methodological issues”. En Eisdorfer C. (Ed), Annual review of gerontology and geriatrics, Vol. 2, pp. 345-382, Springer, New York.

Gerstorf, D., Lövdén, M., Röcke, C., Smith, J. y Lindenberger, U. (2007), "Well-being affects changes in perceptual speed in advanced old age: longitudinal evidence for a dynamic link”, Developmental Psychology Copyright 2007 by the American Psychological Association. Vol. 43, nº 3, pp. 705-718.

Gfellner, B. M. (1989), "Perceptions of health, abilities, and life satisfaction among very old adults”. Perceptual and Motor Skills, 68(1), 203-209. Citado en Inga, J. y Vara, A. (2006), "Factores asociados a la satisfacción de vida en adultos mayores de 60 años en Lima-Perú”, Univ. Psychol, Bogotá. Vol. 5 nº 3, pp. 475-485.

Goleman, D. (2003), Emociones destructivas, Ediciones B Argentina S.A.

Headey, B. y Wearing, A. (1989), "Personality, life events and subjective well being, toward a dynamic Equilibrium model”, Journal of Personality and Social Psychology. Vol. 57, nº 4, pp. 731-739.

Hernández, P. (2006), “Inteligencia emocional y moldes mentales en la explicación del bienestar subjetivo”, Ansiedad y Estrés. Vol. 12, (2-3), pp. 439-462. 
Horley, J. (1984), “Life Satisfaction, happiness, and morale: two problems with the use of subjective web-being indicators”, Gerontologist. Vol. 24, pp. 124-127.

Inga, J. y Vara, A. (2006), "Factores asociados a la satisfacción de vida en adultos mayores de 60 años en Lima-Perú”, Univ. Psychol. Bogotá. Vol. 5, $\mathrm{n}^{\circ} 3$, pp. 475-485.

Instituto Nacional de Estadísticas y Comisión Económica para América Latina y el Caribe (2000), "Chile: Proyecciones y Estimaciones de Población”. Total País. 1950 - 2050.

Lee, S. (2008), Ingresos Económicos: ¿Influye en la Satisfacción Vital y Autoestima de las Personas? Un estudio exploratorio con una muestra de la ciudad de Arica. Tesis de Magíster en Psicología Social, Universidad de Tarapacá, Arica, Chile.

Little, B. (1999), "Personality and Motivation: Personal Action and the Conative Evolution”. En: En Pervin, L. y John, O. (Eds.), Handbook of Personality. Theory and Research.. 2nd ed, pp. 501-524, Guilford Press, New York.

López J. y Crespo, M. (2007), “Intervenciones con cuidadores de familiares mayores dependientes: una revisión”, Psicothema. Vol. 19, n 1, pp. $72-80$.

Mella, R., González, L., D’Appolonio, J., Maldonado, I., Fuenzalida, A., y Díaz, A. (2004), "Factores Asociados al Bienestar Subjetivo en el Adulto Mayor”, Psykhe. Vol.13, nº 1, pp. 79-89.

Moyano, E. y Ramos, N. (2007), "Bienestar subjetivo: Midiendo satisfacción vital, felicidad y salud en la población chilena de la Región del Maule”,,Revista electrónica Universum, Vol. 22.

Palomera, R. y Brackett, M. (2006), "Frequency of positive affect as a possible mediator between perceived emotional intelligence and life satisfaction”, Ansiedad y estrés, Vol. 12 (2-3), pp. 231-239

Pavot, W., y Diener. E., (1993), “The affective and cognitive contest of self reports measures of subjective well-being”, Social Indicators Research. Vol. 28, pp. 1-20.

Peiró, A. (2001), Condiciones socieconómicas y felicidad de los españoles. Instituto Valenciano de Investigaciones económicas S.A., Valencia.

Pereira, M. (1999), Impacto demográfico del adulto mayor en la actividad nacional. Seminario internacional la tercera edad en el tercer milenio. Un desafío para todos, Talca, Chile. 
Ranzijn, R. (2002), “The potential of older adults to enhance community quality of life: Link between positive psychology and productive aging”. Ageing internacional, Vol. 27 nº 2, pp. 33-55.

Richards, L. (1989), “The precarious survival and hard won satisfactions of white single parent families”, Journal of family relations, Vol. 38, n 4 . p. 396.

Rohe, W. y Stegman, M. (1994), “The effects of homeownership on the self esteem, perceived control and life satisfaction of low income people”.,Journal of the American Planning Association, Vol. 60, n 2, p.173.

Ryan, R. y Deci, E. (2001), “On happiness and human potentials: A review of research on hedonic and eudaimonic well-being”, Annual Review of Psychology, Vol. 52, pp. 141-66.

Seligman, M. (2003), La Auténtica Felicidad, Ediciones B, Barcelona.

Seligman, M., y Csikszentmihalyi, M. (2000), "Positive Psychology. An Introduction”, American Psychologist, Vol. 55, n 1, pp. 5-14.

Stearns, C. y Stearns, P. (1986), “Anger: the struggle for emotional control in America's history”. Chicago: University of Chicago Press. Citado en Alcalá, V., Camacho, M., Giner, D. e Ibáñez, E. (2006). “Afectos y género”, Psicothema. Vol. 18, n 1 , pp. 143-148.

Veenhoven, R. (1994), "El estudio de la satisfacción con la vida”, Intervención Psicosocial. $\mathrm{n}^{\circ}$ 3, pp. 87-116. Citado en Blanco, A. y Díaz, D. (2005). “El bienestar social: su concepto y medición”, Psicothema 2005. Vol. 17, n 4, pp. 582-589.

Veenhoven, R. (1984), “Conditions of Happiness”, The Netherlands: Kluwer Academic, Dordrecht.

Wilson, W. (1967), “Correlates of avowed happiness”, Psychological Bulletin. Vol. 67, pp. 294-306.

Zhang, L. (2005), "Prediction of Chinese life satisfaction: Contribution of collective self-esteem”, International Journal of Psychology. Vol. 40, $\mathrm{n}^{\circ} 3$, pp. 189-200.

Zhang, L. y Leung, J. (2002), “Moderating effects of gender and age on the relationship between self-esteem and life satisfaction in mainland Chinese”, International Journal of Psychology. Vol. 37, n 2, pp. 83-91. 